\section{Check for updates}

Cite this: J. Mater. Chem. A, 2021, 9, 15744

Received 5th March 2021

Accepted 14th April 2021

DOI: $10.1039 / \mathrm{d} 1 \mathrm{ta0} 1861 \mathrm{~b}$

rsc.li/materials-a

\title{
The effect of ionomer content in catalyst layers in anion-exchange membrane water electrolyzers prepared with reinforced membranes (Aemion+ $\left.{ }^{\mathrm{TM}}{ }^{\mathrm{T}}\right) \dagger$
}

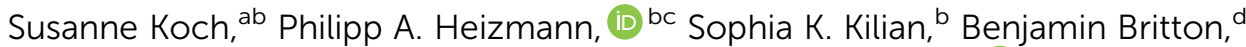 \\ Steven Holdcroft, ${ }^{e}$ Matthias Breitwieser ${ }^{\text {ab }}$ and Severin Vierrath (iD *abc
}

\begin{abstract}
Anion-exchange membrane water electrolyzers (AEMWEs) have seen a significant rise in performance and durability in recent years. However, systematic studies of membrane-electrode assembly parameters such as ionomer and catalyst contents are scarcely available. In this work, we provide an electrochemical and microscopic analysis of ionomer content in anode and cathode catalyst layers to provide insight into their impact on AEMWE performance and stability. The results are based on catalyst-coated membranes (CCMs) using commercially-available, reinforced Aemion+ ${ }^{\mathrm{TM}}$ membranes (lonomr Innovations Inc.) and Aemion+TM ionomer binder in the catalyst layers reaching a performance of $1 \mathrm{~A} \mathrm{~cm}^{-2}$ at a voltage below $2 \mathrm{~V}$. The ionomer content in the cathode catalyst layer was shown to have minimal influence on performance and to allow stable performance in AEMWE using both $10 \mathrm{wt} \%$ and $20 \mathrm{wt} \%$. Varying the ionomer content in the anode catalyst layer resulted in an optimized content of $7 \mathrm{wt} \%$ ionomer, providing a sufficiently low content to avoid mass transport limitation, and sufficiently high content to properly bind the catalyst as observed in a constant current hold stability test. Performance was found to be stable over a range of $0.001-1 \mathrm{M} \mathrm{KOH}$ in the feed solution with slight improvement for higher $\mathrm{KOH}$ contents. This study highlights the importance of balancing the demands of catalyst utilization and mass transport with mechanical and hydrolytic stability of the catalyst layers.
\end{abstract}

\section{Introduction}

Green hydrogen, produced via electrolysis using energy from renewable sources, is a key to address the challenges of climate change and to achieve the goals of international climate agreements. ${ }^{1}$ The largest sources of green hydrogen production today are water electrolysis based on proton-exchange membranes (PEMWE), operating with pure water, and on historically widespread alkaline water electrolysis (AWE), operating with strongly alkaline liquid electrolyte (such as $6 \mathrm{M}$ $\left.\mathrm{KOH}^{2}\right) .{ }^{1,3}$ The development of anion-exchange polymers and membranes (AEMs) now offers the possibility of combining the advantages of both PEM and alkaline technologies. Membranebased electrolyzer systems with alkaline media are expected to

${ }^{a}$ Hahn-Schickard, Georges-Koehler-Allee 103, 79110 Freiburg, Germany. E-mail: severin.vierrath@imtek.uni-freiburg.de

${ }^{b}$ Electrochemical Energy Systems, IMTEK - Department of Microsystems Engineering, University of Freiburg, Georges-Koehler-Allee 103, 79110 Freiburg, Germany 'University of Freiburg, Institute and FIT - Freiburg Center for Interactive Materials and Bioinspired Technologies, Georges-Köhler-Allee 105, 79110 Freiburg, Germany ${ }^{d}$ Ionomr Innovations Inc., 111-2386 East Mall, Vancouver, BC V6T 1Z3, Canada ${ }^{e}$ Department of Chemistry, Simon Fraser University, 8888 University Drive, Burnaby, BC, V5A 1S6, Canada

$\dagger$ Electronic supplementary information (ESI) available. See DOI: 10.1039/d1ta01861b be capable of employing non-noble, earth-abundant catalyst materials avoiding the use of their scarce and expensive counterparts in acidic systems, while still allowing operation at high current densities and differential pressures., ${ }^{3,32}$ The importance of AEMs is further underscored as they provide the foundation for other applications such as electrochemical carbon dioxide reduction which employs similar catalysts and a mildly alkaline anode environment. ${ }^{4}$ Many emerging AEMs are based on fluorine-free, hydrocarbon polymers with the potential advantage of lower synthesis cost compared to incumbent fluorinebased membrane chemistry. ${ }^{5}$ Some hydrocarbon PEM and AEM membranes (such as sulfonated poly(phenylene sulfone), SPPS $^{6}$ or Tokuyama $201^{7}$ ) have shown a promising reduction of the gas crossover compared to the standard fluorine-containing PEM (e.g. Nafion) or alkaline electrolysis diaphragms. ${ }^{2}$ This may facilitate operation at differential pressures and utilizing thinner membranes., ${ }^{2,6}$ While AEM water electrolyzers are an emerging technology, ${ }^{8,9}$ wide-spread use is still limited because of issues of stability of the membranes and constituent polymers, effects critically exacerbated at the elevated temperatures required for efficient electrocatalysis. ${ }^{3,32}$ For membranes, mitigation strategies addressing mechanical and hydrolytic stability include the adoption of woven or non-woven fiber reinforcements of the membrane. Reinforcement reduces swelling of the materials in water and greatly improves handling of the 
membranes. The issue of inherent stability of the constituent hydroxide-conducting polymer is being addressed by newly developed anion-exchange polymers, which show significantly increased chemical stability in alkaline environments at elevated temperatures and thus indicate the potential of stable operation in electrochemical applications running for tens of thousands of hours. ${ }^{5,10-14}$

Electrodes for AEMWE are typically either applied to a transport layer (i.e., catalyst-coated substrate, CCS) or directly onto the membrane (i.e., catalyst-coated membrane, CCM) or a combination of both. ${ }^{3,15}$ While both approaches can significantly differ in performance, neither has been established as a preferred method. This uncertainty is likely due to varying conditions employed, which depend on the catalyst and its loading (e.g. $<1 \mathrm{mg} \mathrm{cm}^{-2}$ for $\mathrm{Pt}^{16}$ in contrast to $>5 \mathrm{mg} \mathrm{cm}^{-2}$ for non-noble $\left.\mathrm{CuCoO}_{x}{ }^{17}\right)$, and on the polymeric binder in the catalyst layers (e.g. PTFE, ${ }^{17}$ Nafion $^{18}$ or anion-exchange polymer $\left.{ }^{16,19}\right)$. Ito et al. concluded that CCMs provided the best mass transport properties, but the catalyst layer stability on the anode side was insufficient and thus a CCS approach, utilizing PTFE as binder, was more successful. ${ }^{20}$

With the emergence of more stable ion-conducting polymers, this study focuses on the investigation of CCMs utilizing the imidazolium-based Aemion+TM (Ionomr Innovations Inc.) both as binder in the catalyst layer and as a reinforced membrane. ${ }^{5}$ Reinforcement of the hydrocarbon-based polymer membrane $(25 \mu \mathrm{m}$ thick) reduces swelling and increases mechanical stability of the membrane. Commercially available noble metal catalysts, $\mathrm{Pt} / \mathrm{C}$ and $\mathrm{IrO}_{x}$, were used to focus on developing electrodes. The Aemion+ content in the catalyst layers was varied to examine catalyst layer homogeneity and stability and its effect on electrolytic performance.

\section{Experimental}

\section{Materials}

$25 \mu \mathrm{m}$ reinforced anion-exchange membrane (AF2-HLE7-25-X) was provided by Ionomr Innovations Inc., which includes a porous reinforcement layer infiltrated with Aemion+ ionomer. Aemion+ anion-exchange ionomer (AP2-HNN6-00-X, Ionomr Innovations Inc.) was used as the catalyst layer binder as described below. $\mathrm{IrO}_{x}$ powder (Premion, 99.99\%, Alfa Aesar) was used as anode catalyst and $\mathrm{Pt} / \mathrm{C}$ (60 wt\%, Greenerity) as cathode catalyst. Ethanol (EtOH, 99.5\%, Ph. Eur., extra pure) and 2propanol (IPA, 70\%, pure) from Carl Roth GmbH + Co. KG and regular laboratory grade deionized water were used.

\section{CCM fabrication}

CCMs were fabricated by spray coating catalyst layers directly onto the as-delivered mixed iodide-chloride-form of the commercial Aemion+ membranes.

Inks were fabricated adapting a procedure reported by Fortin et al. ${ }^{16}$ developed for commercially available Aemion membranes. Here, inks were prepared by first mixing a stock solution of Aemion+ ionomer in a $10: 1$ ethanol : $\mathrm{DI}-\mathrm{H}_{2} \mathrm{O}$ mixture $\mathrm{v} / \mathrm{v}$, where a stable solution was obtained after stirring the mixture at $50{ }^{\circ} \mathrm{C}$ overnight. Catalyst inks were fabricated by adding the required amount of water to the catalyst powder, stirring at room temperature for $5 \mathrm{~min}$ to ensure that the powder was wetted. IPA was added in a $\mathrm{H}_{2} \mathrm{O}$ : IPA ratio of $1: 1 \mathrm{v} / \mathrm{v}$ with subsequent stirring. The ink was transferred to an ultrasonic bath filled with ice water and sonicated while the necessary amount of ionomer stock solution was added drop-wise. The ink for both catalyst layers contained $2 \mathrm{wt} \%$ solids in liquid, while the amount of ionomer in solids was varied $(10 \mathrm{wt} \%$ and $20 \mathrm{wt} \%$ for the cathode and $4 \mathrm{wt} \%, 7 \mathrm{wt} \%, 10 \mathrm{wt} \%$, and $15 \mathrm{wt} \%$ for the anode).

After mixing, and prior to spray coating, inks were stirred overnight and sonicated using a sonication horn for 40 minutes in an ice bath. Commercial $25 \mu \mathrm{m}$ reinforced Aemion+ membranes were fixed between two $1.5 \mathrm{~mm}$ thick polymethylmethacrylate templates with superimposed openings of $4 \mathrm{~cm}^{2}$ on each side.

The spray coating procedure was adapted from Klose et al. ${ }^{6}$ where details can be found. An ultrasonic spray coater (SNR 300, Sonocell) was used with a $60 \mathrm{kHz}$ ultrasonic nozzle (Sonaer NS60K Atomizer) set to $90 \%$ power. Thin metal plates of Nickelplated steel $\left(1 \mathrm{~mm}\right.$ Nickelband, Akkuparts24.de) of $2 \mathrm{~cm}^{2}$ area were placed next to the membranes and weighed before and multiple times during the spray process to track and evaluate the loading of the catalyst layer. To avoid significant precipitation (especially in the anode catalyst inks), syringes of less than $5 \mathrm{~mL}$ volume were used to supply ink to the spray coater. Furthermore, the ink in the syringe was stirred continuously during the spray process, while the remaining ink was kept in an ultrasonic ice bath until the next refill. After full deposition of the catalyst layer, the membranes were left on the $40{ }^{\circ} \mathrm{C}$ hot plate of the spray coater for at least $30 \mathrm{~min}$ before flipping and depositing the other side or storing at room temperature at least over night before proceeding with ion exchange or measurements, to ensure full evaporation of the solvents. The loading was approximately $1 \mathrm{mg}_{\mathrm{Ir}} / \mathrm{cm}^{2}$ on the anode side (as determined by X-ray fluorescence spectroscopy, see Fig. $\mathrm{S} 1 \dagger$ ) and $0.5 \mathrm{mg}$ $\mathrm{cm}^{-2} \mathrm{Pt}$ on the cathode side.

Ion exchange of the ionomer and membrane shipped in the mixed iodide-chloride form was carried out by submersing the CCM in $1 \mathrm{M}$ potassium hydroxide (KOH, ACS reagent, $\geq 85 \%$, pellets, Sigma Aldrich mixed with deionized water) for 48 hours, exchanging the $\mathrm{KOH}$ solution with fresh $\mathrm{KOH}$ solution after 24 hours. The membranes are yellow upon delivery but become almost colorless after a few minutes in $\mathrm{KOH}$, retaining only a slight hint of yellow after the two-day exchange. No measurements were taken to ensure that the membranes and ionomer are fully in hydroxide form, a small amount of retained iodide is expected even after two days in $\mathrm{KOH}$, and carbonates may form whenever the materials are in contact with air after ion exchange. ${ }^{5,21,22}$ To improve handling, membranes were fixed in PTFE templates with superimposed openings exposing the catalyst layers (see Fig. S2 $\dagger$ ), which kept the CCMs flat and avoids their curling, which may otherwise hinder successful assembly of the MEA. 


\section{Electrochemical measurements}

The CCMs were assembled wet, directly after removing them from $\mathrm{KOH}$ and gently wiping off excess liquid. The comparably flat, sprayed CCMs start curling during the ion-exchange in $\mathrm{KOH}$ and retain particularly curled edges due to retention of liquid, which is mitigated by using templates during the ionexchange process (Fig. S2 $\dagger$ ). To avoid drying of the CCMs and minimize the formation of the carbonate form of the ionomer, assembly was done quickly, immediately before measurements were initiated and feed solution was circulated. Since the CCMs were assembled wet after immersing in $1 \mathrm{M} \mathrm{KOH}$ for ionexchange, a small amount of $\mathrm{KOH}$ was introduced into the system. For the higher concentrations this amount is most probably negligible. However, in the case of water as feed solution the amount of introduced $\mathrm{KOH}$ has to be considered. For that case a $\mathrm{KOH}$ concentration of below $0.001 \mathrm{M}$ was estimated from the ratio of the feed solution volume $(2 \mathrm{~L})$ and a $1 \mathrm{~mm}$ thick film covering both sides of the $4 \mathrm{~cm}^{2} \mathrm{CCM}(800$ $\mu \mathrm{L})$.

As porous transport layer (PTL) on the anode side, Ti-felts (Bekaert, 2GDL40-1.0) with a thickness of $1 \mathrm{~mm}$ were used following cutting and cleaning procedures previously published. ${ }^{23}$ PTLs were $5 \mathrm{~cm}^{2}$ and thus protrude over the $4 \mathrm{~cm}^{2}$ sprayed CCM area. This reduces mechanical strain to the edges of the sprayed area, which were seen to be the most likely area for the formation of pinholes in previous studies using less stable membrane materials. The transport layer on the cathode side was carbon paper with a microporous layer (H24C5, Freudenberg), thickness of $270 \mu \mathrm{m}$, cut to $4 \mathrm{~cm}^{2}$, thus limiting the cell area to $4 \mathrm{~cm}^{2}$. Compression of the CCM in the test cell was high as noted in ref. 23. The Ti PTLs are expected to be rigid and were thus fixed in a $1 \mathrm{~mm}$ PTFE gasket of the same thickness as the PTL. The cathode GDL was fixed in a $150 \mu \mathrm{m}$ PTFE frame and thus compressed by approximately $44 \%$, this value significantly influences the high-frequency resistance of the cell. The PTFE gasket material (PTFE skived film, High-tech-flon) was cut to include superimposed openings of $5 \mathrm{~cm}^{2}$ on the anode and 4 $\mathrm{cm}^{2}$ on the cathode side to fit the transport layers exactly. The cell assembly was tightened using a torque force of $6 \mathrm{Nm}$.

CCMs were assembled in a custom-built test cell using PEEK endplates, copper current collectors (separated from the feed solution by ethylene propylene diene monomer rubber, EPDM O-rings) and Ti flow fields with a parallel flow pattern, which were sputtered with gold in the flow field area to improve contact resistances. Electrochemical measurements were performed using a Scribner 857 potentiostat and a BioLogic VSP300 with two $10 \mathrm{~A} / 5 \mathrm{~V}$ amplifiers, which were confirmed to have comparable results by testing multiple stable-performance cells with both systems. Measurements were performed at $60{ }^{\circ} \mathrm{C}$, circulating defined concentrations of below $0.001,0.1$, 0.5, $1 \mathrm{M} \mathrm{KOH}$ solution as specified, (all were mixed from $\mathrm{KOH}$ pellets dissolved in deionized water, which was de-gassed by bubbling nitrogen gas for at least 1 hour) with $40 \mathrm{~mL} \mathrm{~min}{ }^{-1}$ after pre-heating in a bath thermostat. To equalize the concentration between the test cell and the test bench system at the desired concentration, the feed solution was circulated through the cell for at least $1 \mathrm{~h}$ before the first polarization curve was run. As a quick performance and short-circuit-check the cell was tested using several potentiostatically controlled voltagesteps after at least $30 \mathrm{~min}$ of feed solution circulation and

Table 1 Standard AEMWE measurement protocol including the short-term degradation test

\begin{tabular}{|c|c|c|c|c|}
\hline \# & Step & Ranges & Step time & Description \\
\hline 1 & Polarization curve & $\begin{array}{l}\text { Current density step values } \\
\text { according to the EU } \\
\text { harmonized measurement } \\
\text { protocol }^{24}\end{array}$ & 2 min per point & $\begin{array}{l}100 \mathrm{~s} \text { current hold, final } 10 \% \\
\text { averaged as measured } \\
\text { voltage, } 20 \mathrm{~s} \text { short } \\
\text { galvanostatic EIS, } 100 \mathrm{kHz} \text { to } \\
100 \mathrm{~Hz} \text {, amplitude } 5-10 \% \text { of } \\
\text { current }\end{array}$ \\
\hline 2 & EIS & $\begin{array}{l}25 \mathrm{~mA} \mathrm{~cm}{ }^{-2}, 100 \mathrm{~mA} \mathrm{~cm}^{-2} \\
250 \mathrm{~mA} \mathrm{~cm}^{-2}, 500 \mathrm{~mA} \mathrm{~cm}{ }^{-2} \\
1000 \mathrm{~mA} \mathrm{~cm}^{-2}, 1100 \mathrm{~mA} \\
\mathrm{~cm}^{-2}\end{array}$ & $\begin{array}{l}2 \min +\text { time for } \mathrm{EIS} \approx 6 \\
\mathrm{~min} / \text { point }\end{array}$ & $\begin{array}{l}2 \text { min current hold, } \\
\text { galvanostatic EIS, } 100 \mathrm{kHz} \text { to } \\
100 \mathrm{mHz} \text {, amplitude } 5-10 \% \\
\text { of current }\end{array}$ \\
\hline 3 & Polarization curve & See step \#1 & See step \#1 & See step \#1 \\
\hline 4 & Constant current hold & $1 \mathrm{~A} \mathrm{~cm}^{-2}$ & $5 \mathrm{~h}$ & $\begin{array}{l}\text { One data point per minute, } \\
\text { consisting of } 40 \mathrm{~s} \text { applied } \\
\text { current, final } 10 \% \text { of voltage } \\
\text { response averaged as } \\
\text { measured voltage, } 20 \mathrm{~s} \text { short } \\
\text { galvanostatic EIS at the same } \\
\text { DC current, } 100 \mathrm{kHz} \text { to } \\
100 \mathrm{~Hz}, 5 \% \text { amplitude }\end{array}$ \\
\hline 5 & Polarization curve & See step \#1 & See step \#1 & See step \#1 \\
\hline 6 & Constant current hold & See step \#4 & See step \#4 & See step \#4 \\
\hline 7 & Polarization curve & See step \#1 & See step \#1 & See step \#1 \\
\hline 8 & EIS & See step \#2 & See step \#2 & See step \#2 \\
\hline 9 & Polarization curve & See step \#1 & See step \#1 & See step \#1 \\
\hline
\end{tabular}


a temperature up to $60{ }^{\circ} \mathrm{C}$ was achieved. In this pre-test the cell was kept at $1 \mathrm{~V}$ (to check for short-circuiting due to e.g. puncturing of the membrane during assembly), $1.4 \mathrm{~V}, 1.6 \mathrm{~V}, 2 \mathrm{~V}$ and $2.3 \mathrm{~V}$ for $50 \mathrm{~s}$ each, before the same steps and durations were taken in the other direction $(2.3 \mathrm{~V}, 2 \mathrm{~V}, 1.6 \mathrm{~V}, 1.4 \mathrm{~V})$ and an electrochemical impedance spectroscopy (EIS) measurement was recorded galvanostatically at $100 \mathrm{~mA} \mathrm{~cm} \mathrm{~cm}^{-2}$. The cell was then kept at $0 \mathrm{~A}$ for the remaining time needed to finish $1 \mathrm{~h}$ of feed solution circulation. The measurement protocol after this pre-test is listed in Table 1. Each step was terminated early or skipped if the applied potential would have exceeded $2.3 \mathrm{~V}$. The short EIS measurements during the polarization curves and current holds were used to determine the high-frequency resistance (HFR) from the crossing of the $x$-axis (real part of impedance) in the Nyquist plot at the specified current density. For all samples and all current densities studied here the $x$-axis crossing in the Nyquist plot was in the range of $100 \mathrm{kHz}$ to $100 \mathrm{~Hz}$, typically at or above $10 \mathrm{kHz}$. At low current densities (below $150 \mathrm{~mA} \mathrm{~cm}{ }^{-2}$ ) HFR values are less reliable due to measurement errors and the low amplitudes $(10 \%$ of DC current) used for EIS.

These measurements result in a short-term durability test amounting to $10 \mathrm{~h}$ at $1 \mathrm{~A} \mathrm{~cm}^{-2}$, or until the voltage surpassed $2.3 \mathrm{~V}$ due to strong degradation.

At least three CCMs were fabricated and measured in the electrolyzer for each variation of ionomer content or feed solution, keeping the other parameters constant.

\section{Focused ion beam scanning electron microscopy (FIB/SEM) cross sections}

Cross sections of the catalyst layers were prepared with a focused ion beam scanning electron microscope (FE-SEM, Amber X, Tescan). Samples were milled by $\mathrm{Xe}^{+}$ions at $20 \mathrm{kV}$ acceleration voltage with a current of $20 \mathrm{nA}$, and polished in a FEI Scios 2 DualBeam FIB-SEM using $\mathrm{Ga}^{+}$ions at $20 \mathrm{kV}$ and $100 \mathrm{pA}$. Images of the catalyst layers were acquired at $2 \mathrm{kV}$ acceleration voltage and a working distance of $6 \mathrm{~mm}$, with 100 pA current using an in-column SE detector. Samples were CCMs sprayed as described above and fixed onto standard SEM stubs using carbon pads.

\section{Transmission electron microscopy (TEM)}

The catalyst layer inks were sprayed directly onto TEM grids using one spraying path of the CCM fabrication spray process to ensure an identical composition. This is in contrast to TEM samples typically prepared by dipping the TEM grids into the catalyst-ionomer ink, which is less comparable to sprayed catalyst layers due to a possible variation in ionomer content and distribution on the TEM grids. Especially in the case of the anode inks, which were based on $\mathrm{IrO}_{x}$ without any supporting carbon and with an AEM ionomer in this study, the ink stability was a persistent issue. By preparing samples during the spray coating process of the CCM, it was ensured that the deposited material was identical to that present in the CCM catalyst layers. TEM sample preparation was integrated into a regular CCM spraying procedure as described above in an in situ spraying process: coated TEM grids (copper, carbon film, 3-4 nm, 400 mesh, science services) were placed next to the CCM during the spraying of the anode side for one spraying path and then removed again. This resulted in sufficient particles on the TEM grid, dispersed automatically via the spraying process from an ink identical to the one used for fabrication of CCMs. TEM images were acquired using a Talos L120C TEM with a $\mathrm{LaB}_{6}$ electron source and $120 \mathrm{kV}$ accelerating voltage. The images were acquired using a Ceta Camera (Thermo Scientific) at 1 nA current.

\section{Results and discussion}

CCMs were fabricated using different amounts of anionexchange polymer in the catalyst layers and studied using microscopic and electrochemical techniques. The morphology of anode catalyst particles and layers was studied using TEM and FIB/SEM imaging.

\section{Anode morphology}

Fig. 1 shows transmission electron microscopy (TEM) images of $\mathrm{IrO}_{x}$ catalyst particles with different amounts of ionomer in the spray coating ink. While for the low ionomer content samples determination of ionomer in TEM images is difficult (Fig. 1a and $b$ ), there is a clear film wrapping around the particles for
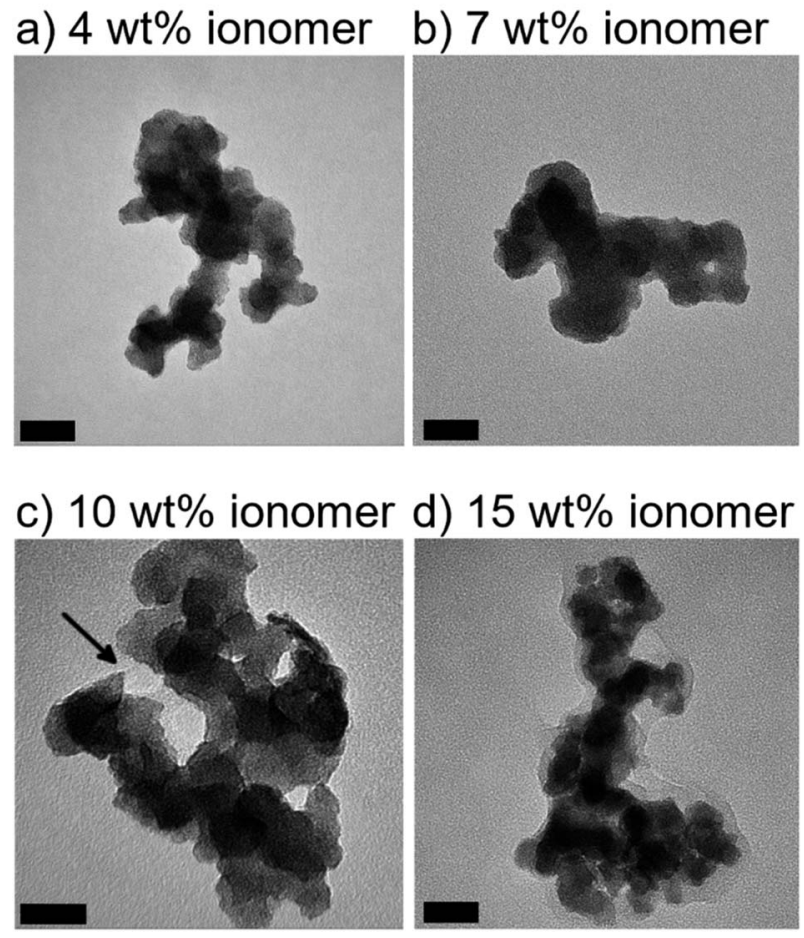

Fig. 1 Representative $\mid \mathrm{rO}_{x}$ catalyst particles with varying amount of anion-exchange ionomer imaged in TEM, scale bars in all images are $20 \mathrm{~nm}$. The samples with 4 wt\% (a) and 7 wt\% (b) show no clearly discernible film-like features around the particles. For $10 \mathrm{wt} \%$ ionomer (c) a faint meniscus is visible in the area indicated by the arrow. For particles with $15 \mathrm{wt} \%$ ionomer (d) a thin, faint film can be seen wrapping around the catalyst particles. 
$15 \mathrm{wt} \%$ ionomer (Fig. 1d) distinguishable from the catalyst powder particles. This film has a thickness of up to $10 \mathrm{~nm}$ and covers most of the particle, while for the $10 \mathrm{wt} \%$ sample only a thin meniscus can be observed, indicated by the arrow in Fig. 1c. The films of ionomer wrapping around the particles may electrically insulate some particles as wt\% of ionomer increases.

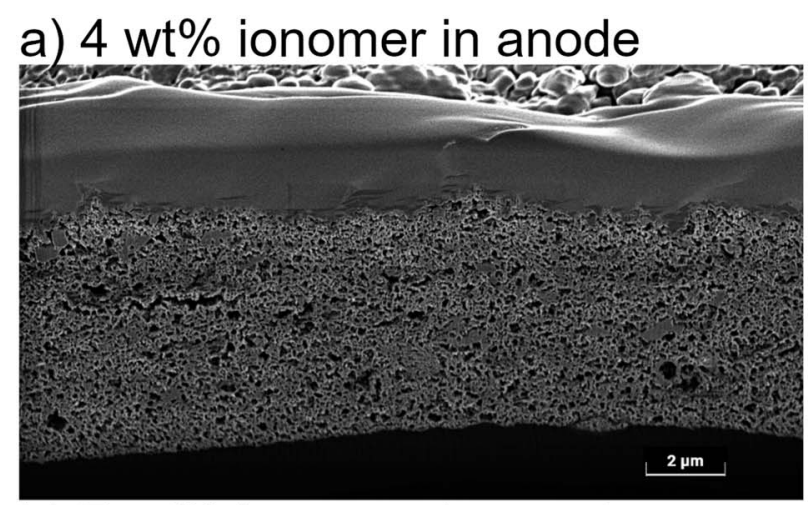

b) $7 \mathrm{wt} \%$ ionomer in anode

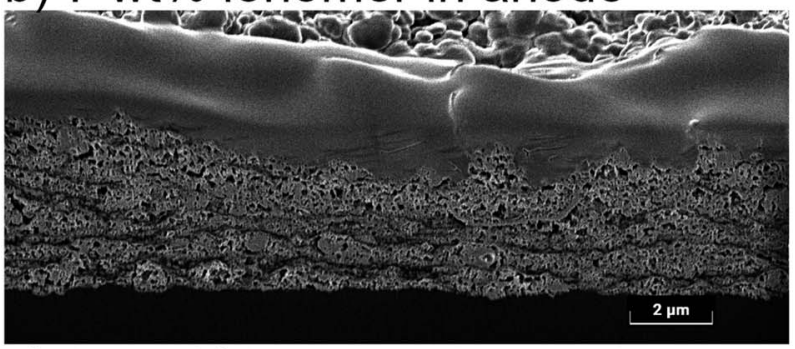

c). $10 \mathrm{wt} \%$ ionomer in anode

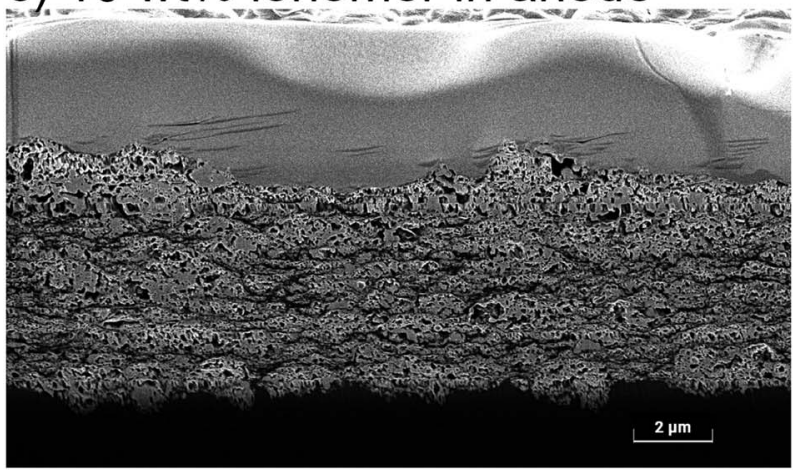

d) $15 \mathrm{wt} \%$ ionomer in anode

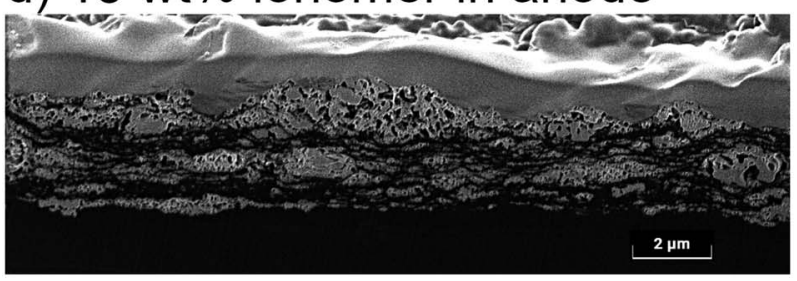

Fig. 2 Representative cross sections of anode catalyst layers containing $\operatorname{IrO}_{x}$ and varying amounts of Aemion+ ionomer prepared by focused ion beam. While a non-linear thickness variation can be observed in the layers, the $15 \mathrm{wt} \%$ ionomer sample clearly shows dark areas within the catalyst layer, potentially indicating ionomer-rich areas and stronger inhomogeneities, which may influence performance.
The variation in ionomer distribution is also visible in cross sections of the catalyst layer prepared by focused ion beam (Fig. 2). In these scanning electron micrographs, regions with high iridium content appear bright, while darker areas are mostly comprised of ionomer - the distinction from void spaces (which also appear dark) is made by apparent texture and form, seen in more detail in magnified SEM images (Fig. S3†). The sample with $15 \mathrm{wt} \%$ shows the most obvious variation, illustrating clear inhomogeneity in the catalyst layer as evidenced by dark regions and vertical streaks, suggesting ionomer-rich areas with the likelihood of reduced porosity and potentially even reduced electrical conductivity. The observed inhomogeneity most likely stems from instability of the ink, which increases in instability with increasing ionomer content. As a consequence, these ionomer-rich areas were not observed in the $4 \mathrm{wt} \%$ sample. A similar variation is observed in the catalyst layer thickness. To assure comparability despite the thickness variation, the catalyst loading was determined by X-ray fluorescence (Fig. S1†), proving that differences in loading were not significant within the margins of error $\left(0.94 \mathrm{mg}_{\text {Ir }} \mathrm{cm}^{-2} \pm 0.1 \mathrm{mg}_{\text {Ir }}\right.$ $\left.\mathrm{cm}^{-2}\right)$.

\section{Electrochemical performance}

Fig. 3a shows the cell polarization of CCMs with $10 \mathrm{wt} \%$ and $20 \mathrm{wt} \%$ ionomer in the cathode layer keeping the ionomer
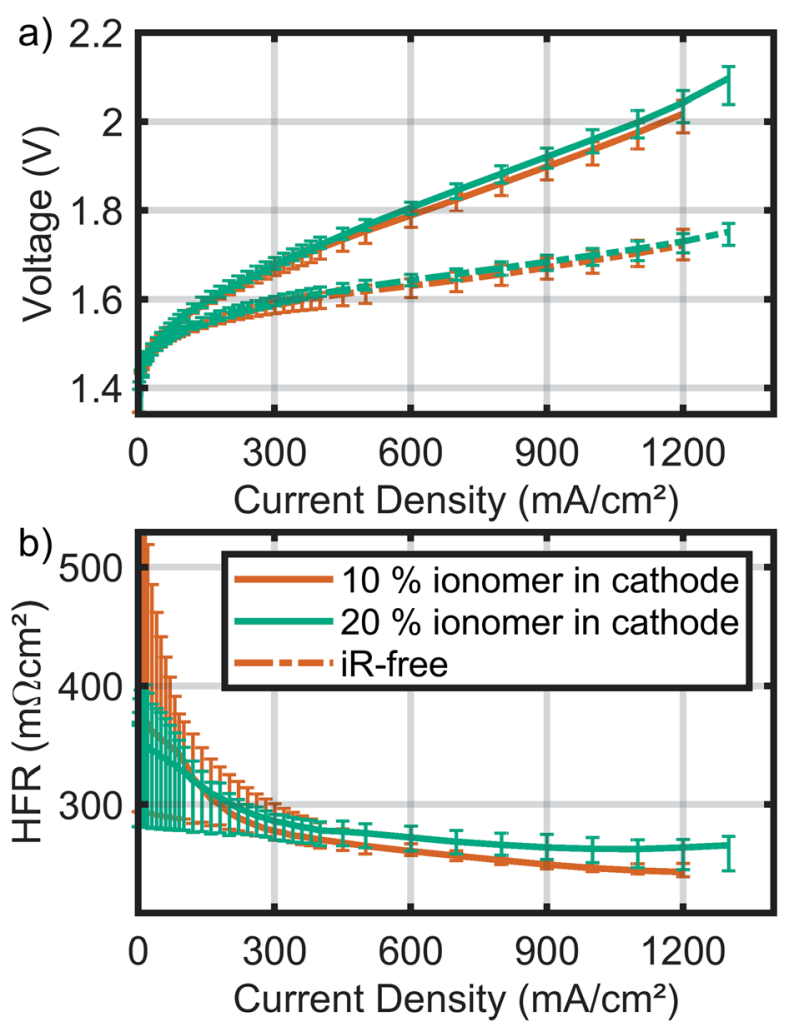

Fig. 3 (a) Cell polarization, iR-free voltage (dashed lines) and (b) high frequency resistance (HFR) for varying amounts of ionomer in the cathode. The error bars correspond to range of performances between two subsequent measurements of two identical samples each (steps 1 and 3 of the protocol in Table 1). All cells were measured in $0.1 \mathrm{M} \mathrm{KOH}$. 
content in the anode CL at $7 \mathrm{wt} \%$ along with the high-frequency resistance (HFR, Fig. 3b) and the resulting iR-free polarization curves (dashed lines). Note that the HFR is not stable across all current densities for the samples studied here, in contrast to the HFR reported in PEMWE. ${ }^{23}$ This effect might be related to membrane humidification, but a detailed investigation is needed to uncover the cause. The reported iR-free performance, which excludes losses due to the membrane and test setup conductivity, is comparable to recently reported results from CCSs based on Aemion membranes measured under the same conditions $\left(\sim 1 \mathrm{~A} \mathrm{~cm}^{-2}\right.$ at $1.68 \mathrm{~V}$ iR-free voltage in $\left.0.1 \mathrm{M} \mathrm{KOH}\right){ }^{16}$ CCMs fabricated in the present study, however, contain less than half of the $\mathrm{IrO}_{x}$ loading as used in the work of Fortin et al. ${ }^{16}$ achieving these same performances, which indicates enhanced catalyst utilization.

Comparing the polarization data of the CCMs with different ionomer contents in the cathode, a similar polarization behaviour and HFR can be observed lying in the range of batch reproducibility (error bars, all data shown in Fig. S4†). Thus, the ionomer content in the cathode has only a small effect on the overall performance of these CCMs. In contrast, recent reports show improved performance for cathode catalyst layers containing $10 \mathrm{wt} \%$ ionomer compared to higher ionomer contents. ${ }^{19,25}$ This is in agreement with a slightly better performance for the $10 \mathrm{wt} \%$ ionomer CCM observed in the present work, but the effect of cathode ionomer content variation is less significant here, likely due to the CCM architecture and different materials employed.

\section{Varied ionomer content in anode catalyst layer}

To observe the influence of ionomer in the anode, the ink composition was varied to include $4 \mathrm{wt} \%, 7 \mathrm{wt} \%, 10 \mathrm{wt} \%$, and $15 \mathrm{wt} \%$ of ionomer in solids. Polarization curves and iR-free curves, as well as the HFR for all anode variations are displayed in Fig. 4. Multiple cells were fabricated for each variation in several spray coating batches and tested in the electrolyzer (all data is shown in Fig. $\mathrm{S} 5 \dagger$ ) to determine cell reproducibility. The observed sample-to-sample variation in Fig. S5 and S4† and the error bars shown in Fig. 3 is believed to be due to instability of the anode ink. While reproducibility is greatly improved by stirring the ink during spray coating, CCMs for all different ionomer contents retained a reproducibility range in performance and - uncorrelated to this - anode catalyst loading. The loading of the $\mathrm{IrO}_{x}$ catalyst was measured post-fabrication using X-ray fluorescence (see Fig. S1 $\dagger$ ). For a consistent comparison, Fig. 4 shows CCMs with similar loading of $0.95 \mathrm{mg}_{\text {Ir }} \mathrm{cm}^{-2} \pm$ $0.04 \mathrm{mg}_{\text {Ir }} \mathrm{cm}^{-2}$.

CCMs with high ionomer content of $15 \mathrm{wt} \%$ in the anode catalyst layer yield an overall lower performance in the kinetic, ohmic and mass transport regions. Samples of lower ionomer contents of $4 \mathrm{wt} \%$ and $7 \mathrm{wt} \%$ are superior in $V-I$ performance and comparable to each other, with the $10 \mathrm{wt} \%$ ionomer CCM lying in between. However, the variations between $4 \mathrm{wt} \%$, $7 \mathrm{wt} \%$, and $10 \mathrm{wt} \%$ ionomer CCMs are smaller than reproducibility margins (as shown in Fig. S5†), therefore a reproducible,
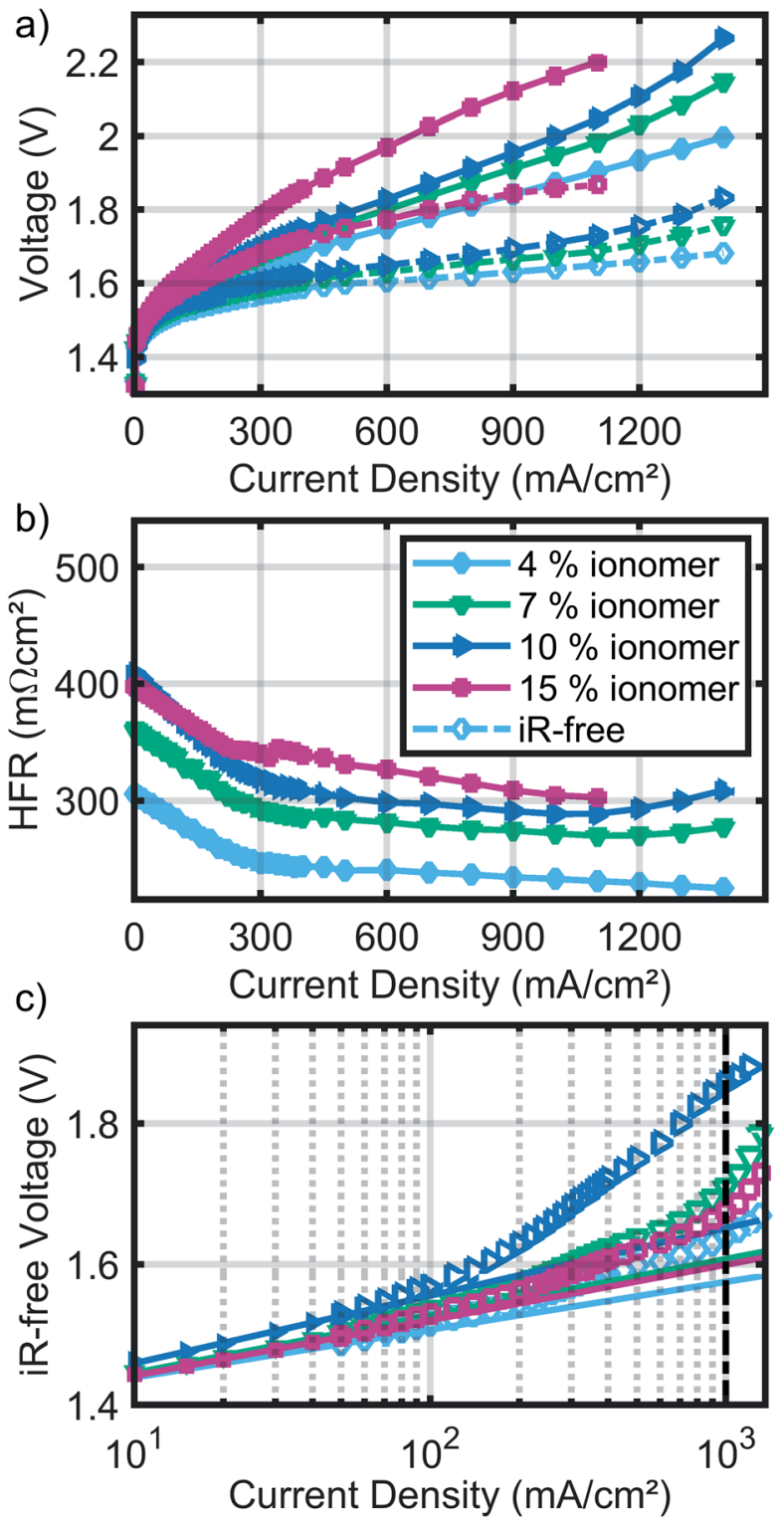

Fig. 4 (a) Cell polarization, iR-free voltage (dashed lines, empty markers) (b) high frequency resistance (HFR) and (c) iR-compensated voltage with a linear fit to the low current density region for varying ionomer content in the anode layer at a low average loading error $\left(0.95 \mathrm{mg}_{\mathrm{rr}} \mathrm{cm}^{-2} \pm 0.04 \mathrm{mg}_{\mathrm{lr}} \mathrm{cm}^{-2}\right.$ ), shown for one measured sample of each variation. The measurements correspond to step 1 in the protocol listed in Table 1. All cells were measured in $0.1 \mathrm{M} \mathrm{KOH}$.

significant deterioration of electrolyzer performance for high ionomer content is only identified for $15 \mathrm{wt} \%$ ionomer content.

For all measured samples with varying anode ionomer content, the HFR was extracted at $400 \mathrm{~mA} \mathrm{~cm}{ }^{-2}$ and $1 \mathrm{~A} \mathrm{~cm}^{-2}$ (Fig. 5a) and a linear fit to the iR-compensated voltage response in the low current density region between 10 and $90 \mathrm{~mA} \mathrm{~cm}^{-2}$ was performed as shown in Fig. 4c. At low current densities the influence of mass transport and hydroxide transport are deemed negligible, ${ }^{26}$ which is used here to extract the mass transport and hydroxide conductivity overpotential at $1 \mathrm{~A} \mathrm{~cm}^{-2}$ (Fig. 5b). Values are the median of all measured samples, while 

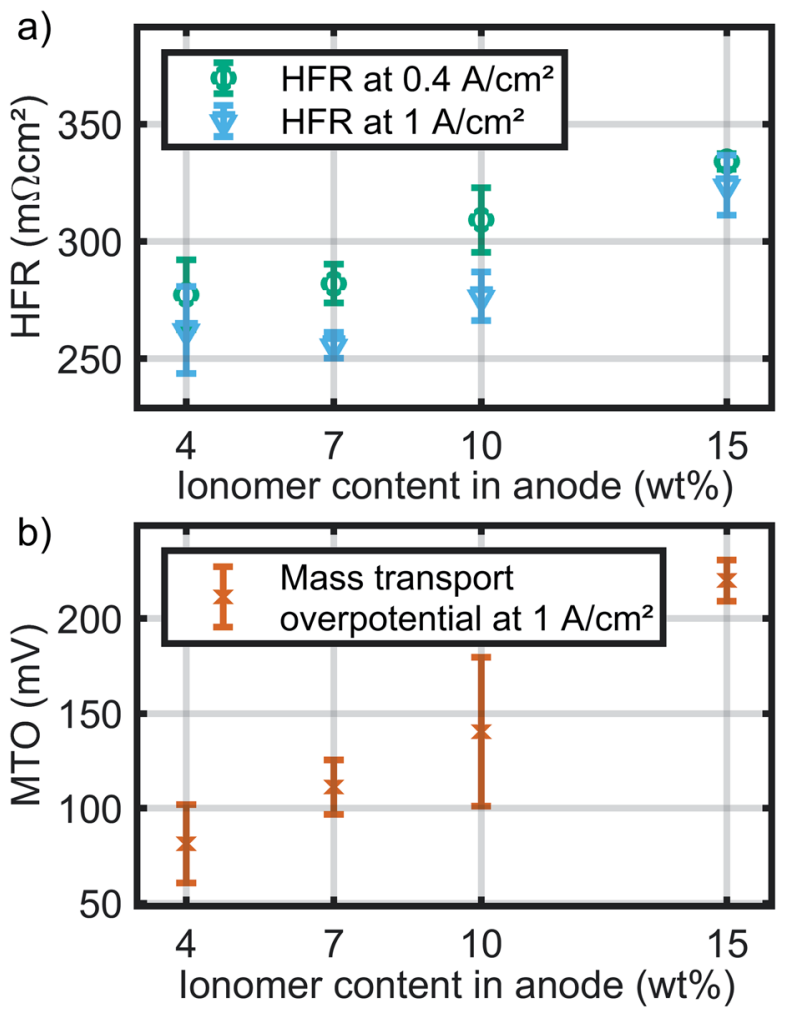

Fig. 5 Performance parameters for all tested ionomer contents in the anode catalyst layer: (a) high frequency resistance extracted from polarization curves at two fixed current densities and (b) mass transport overpotential determined at $1.2 \mathrm{~A} \mathrm{~cm}^{-2}$ by subtracting the kinetic contribution estimated through a linear fit of the $\mathrm{iR}$-free polarization curves in the current density range of $10-80 \mathrm{~mA} \mathrm{~cm}^{-2}$. Markers show the median of at least three cells of each variation and error bars are the average distance from the median.

error bars indicate the average distance from the median as appropriate for a small number of observations. ${ }^{27}$ In the range around $40 \mathrm{~mA} \mathrm{~cm}{ }^{-2}$ where a primarily kinetic response can be expected a trend is seen in Fig. 4c pointing to higher overpotentials for increasing ionomer content, which may indicate a decreased number of active catalyst particles for the higher contents. However, the analysis of the kinetic region is distorted by the crossover and shunt current contributions in the very low current range and by mass transport in the higher current range. The current-voltage response in this region also contains a complex overlap of contributions from the oxygen evolution reaction and hydrogen evolution reaction and their separation is not trivial. To achieve a more thorough and complete understanding of the kinetic region, overlapping effects need to be excluded, so other measurements such as vapor-fed water electrolysis should be employed, as recently reported for protonexchange membrane water electrolyzers by Schuler et al. ${ }^{\mathbf{2 8}}$

An increasing trend is observed for all loss factors when the ionomer content is increased, with the lowest overall performance for the $15 \mathrm{wt} \%$ sample. Values for $4 \mathrm{wt} \%, 7 \mathrm{wt} \%$ and $10 \mathrm{wt} \%$ in some cases overlap within their errors. This overall trend is comparable to recent observations made by Huang et al. using a different set of materials and a range of ionomer contents of $15 \mathrm{wt} \%$ to $45 \mathrm{wt} \%$, where the lowest ionomer content also showed improved performance. ${ }^{25}$

The high frequency resistance at moderate and higher current densities as shown in Fig. 5a increases from around 250 to $340 \mathrm{~m} \Omega \mathrm{cm}^{2}$ with increasing ionomer content. The HFR consists of the electrical resistance of the test setup, GDLs and catalyst layer as well as the ionic resistance of the membrane. As only the catalyst layer composition was altered across the samples, the increasing HFR reflects an increasing electrical resistance in the catalyst layer. The cause most probably lies in increasing electrical isolation of catalyst particles due to increasing ionomer films, which is in line with morphological observations (Fig. 1 and 2).

The extracted mass transport overpotential increases from around $100 \mathrm{mV}$ for the lowest ionomer content to above $200 \mathrm{mV}$ for the $15 \mathrm{wt} \%$ sample. In light of the observations made from TEM and FIB/SEM images this could be explained by reduced gas diffusion due to ionomer covering the catalyst and pores filled with ionomer, reducing overall porosity. A more pronounced low frequency semi-circle in the impedance spectrum of the $15 \mathrm{wt} \%$ sample supports this explanation (see Fig. S7 $\dagger$ ). At the same time the high ionomer content may insulate some catalyst particles so far that the electrochemically active surface area is reduced, which in turn would lead to a higher local current at the catalyst and thus higher mass transport losses at the catalyst.

\section{Anode stability under constant current}

The summarized results of constant current tests for cells with varying ionomer content in the anode are shown in Fig. 6, while mean potential evolutions are shown in Fig. S6. $\dagger$ The $4 \mathrm{wt} \%$ ionomer anode has a higher median value and larger error for the initial voltage than the $7 \mathrm{wt} \%$ sample, which is explained by a time delay of around 2 hours between the initial polarization curve and the start of the degradation test, where electrochemical impedance spectroscopy measurements were performed. The same can be observed for the voltage increase during a 5 hour current hold at $1 \mathrm{~A} \mathrm{~cm}^{-2}$. Likewise, in the case of the $15 \mathrm{wt} \%$ ionomer anode a notable gap is seen in initial voltage (as expected from performance parameters), but the degradation slope is unexpectedly similar to those of the lower ionomer contents, overlapping error ranges with the $10 \mathrm{wt} \%$ and $4 \mathrm{wt} \%$ samples. A potential explanation for the lack of a clear trend is the instability of the anode catalyst layer causing the voltage increase. In this process, the ionomer is potentially dislodged due to the continuous circulation of feed solution at $60{ }^{\circ} \mathrm{C}$, leading to a loss of catalyst particles. Loading determination before and after measurements using XRF (Fig. S8 †) confirms this finding, while a degradation of the membrane is unlikely due to the comparative stability of the high frequency resistance (Fig. 6c). Electrochemical impedance spectroscopy was measured before and after the degradation test and is shown in Fig. $\mathrm{S} 7 \dagger$ at three DC current densities $\left(100 \mathrm{~mA} \mathrm{~cm}{ }^{-2}\right.$, $500 \mathrm{~mA} \mathrm{~cm}^{-2}$ and $1 \mathrm{~A} \mathrm{~cm}^{-2}$ ) for one cell of all variations of the ionomer content in the anode catalyst layer (step 2 and step 8 of the measurement protocol in Table 1). The cells are identical 

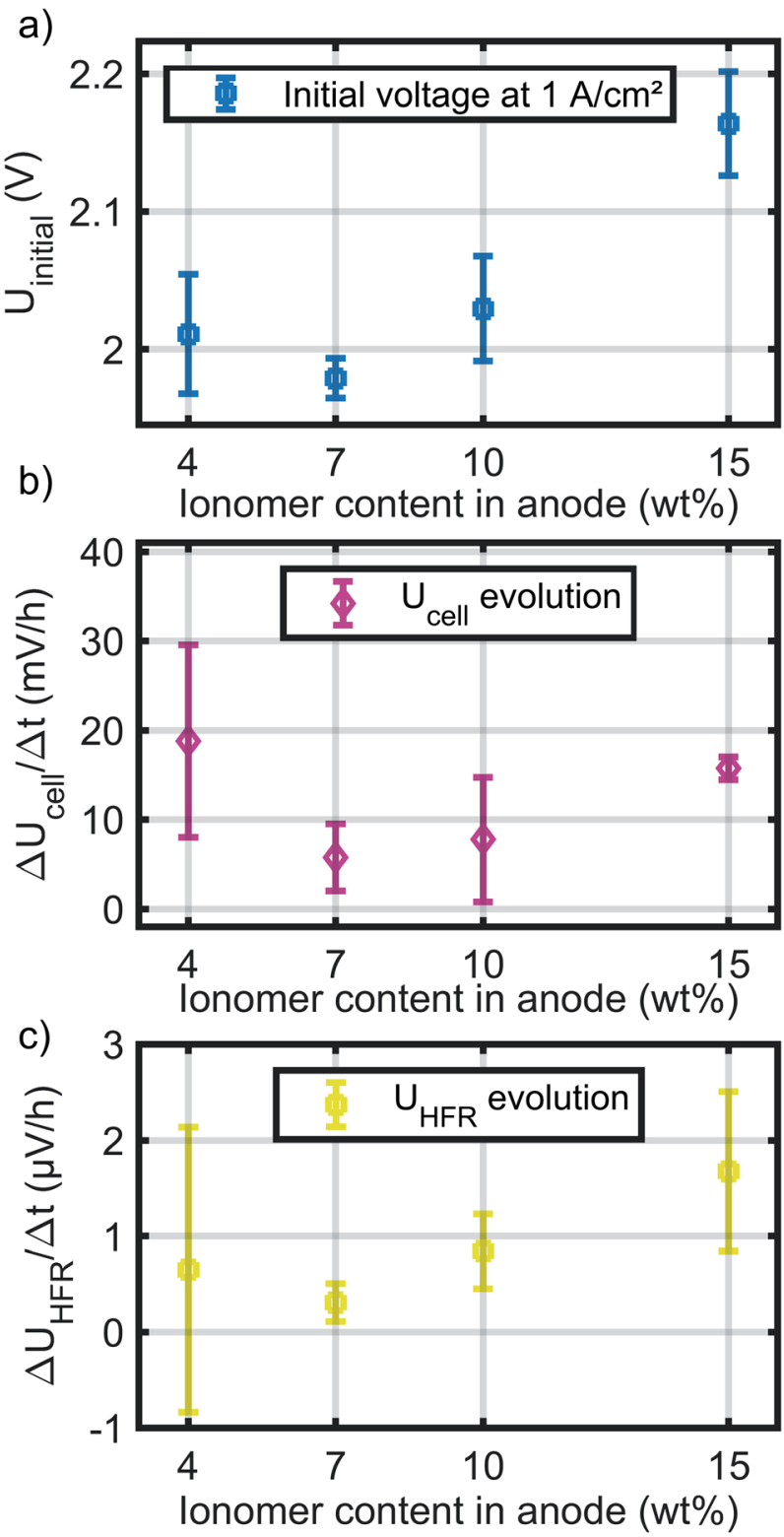

Fig. 6 Degradation parameters for all tested ionomer contents in the anode catalyst layer: (a) initial voltage at the current density of $1 \mathrm{~A}$ $\mathrm{cm}^{-2}$, (b) evolution of the cell voltage over a $10 \mathrm{~h}$ current hold and (c) the contribution of the evolution of the high frequency resistance to the voltage calculated using Ohm's law. The slopes of the evolutions were extracted from linear fits to the measured cell response during the current hold.

with the ones of which polarization traces are shown in the main text. The Nyquist plots illustrate minimal changes in the HFR upon degradation while a significant difference is seen between ionomer content variations. No membrane degradation was observed for the samples after disassembling the cell. Fortin et al. also linked short-term degradation observed for Aemion to instabilities of the catalyst layers, which could be mitigated by a change in ionomer and ionomer content. ${ }^{16}$ Continuous constant operation at high current densities is a standard stress test for water electrolysis. ${ }^{29}$ To provoke a high degradation rate, a two times higher current density was chosen in this work in comparison to the study by Fortin et al. (1.0 A $\mathrm{cm}^{-2}$ vs. $0.5 \mathrm{~A} \mathrm{~cm}^{-2}$ ). In spite of this increase in current density and a three times lower anode catalyst loading employed here, we find comparable degradation rates for the $7 \mathrm{wt} \%$ and $10 \mathrm{wt} \%$ samples of below $10 \mathrm{mV} \mathrm{h}^{-1}$, which is a promising improvement of cell stability. This suggests that a careful balance has to be chosen to develop an optimized CCM: the ionomer content should be low enough to provide adequate performance, layer homogeneity and thus a low mass transport overpotential, HFR and low kinetic losses, but high enough to maintain catalyst layer stability.

\section{Feed solution concentration variation}

Fig. 7 shows polarization curves and high frequency resistances for four samples fabricated within one batch (on the same day using the same spraying inks, parameters and operator), which were measured in feed solutions of 0.001 to $1 \mathrm{M} \mathrm{KOH}$. The samples were fabricated with the optimized ionomer contents of $7 \mathrm{wt} \%$ in the anode and $20 \mathrm{wt} \%$ in the cathode, choosing a low ionomer content to yield stable anodes. It should,
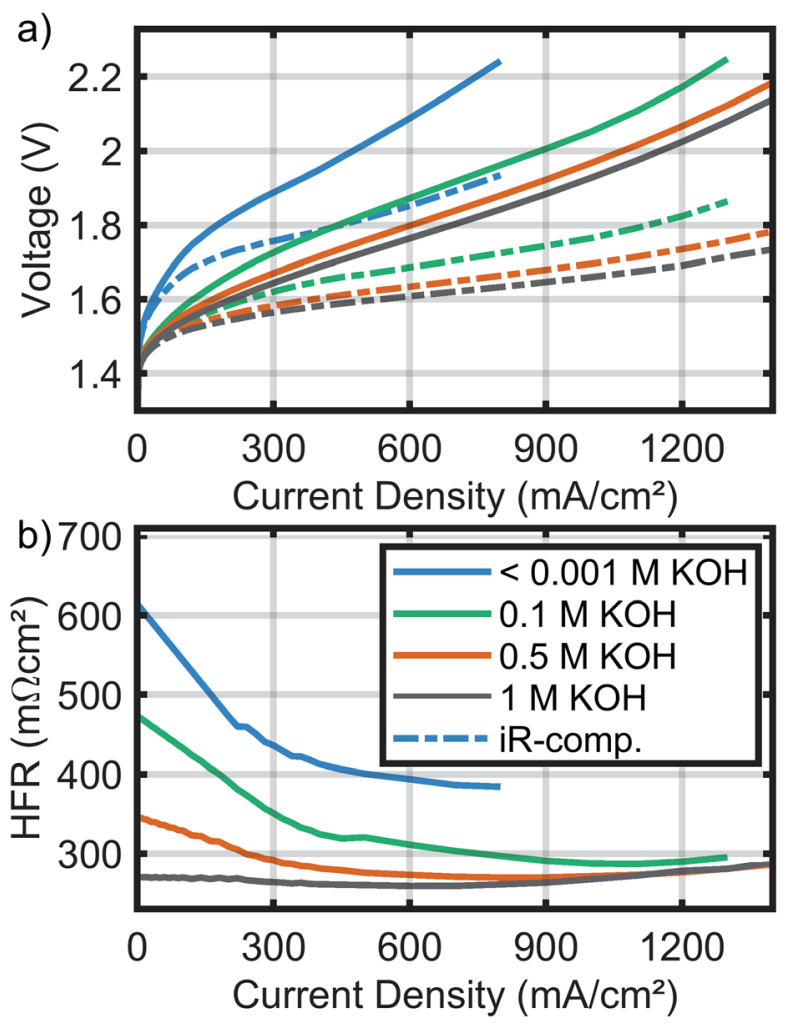

Fig. 7 (a) Cell polarization, iR-free voltage response (dashed lines) and (b) HFR over current density of four cells using different molarities of $\mathrm{KOH}$ in water as the electrolyzer feed solution. After ion-exchange in $1 \mathrm{M} \mathrm{KOH}$ all cells were assembled quickly while fully wet and circulated with the feed solution for at least $1 \mathrm{~h}$ before measuring. The lowest concentration sample was measured with DI-water as feed-solution, which results in the estimated $<0.001 \mathrm{M} \mathrm{KOH}$ concentration due to a small amount of $1 \mathrm{M} \mathrm{KOH}$ introduced to the system from the wet CCM. 
however, be noted that the batch shown here for the molarity variation was one on the lower performance spectrum due to the observed instability of the catalyst inks, which also serves as the explanation why only samples from one spraying batch are shown here (all results of $\mathrm{KOH}$ variations can be found in Fig. S9†).

The feed solution was varied between $1 \mathrm{M}, 0.5 \mathrm{M} \mathrm{KOH}$ and $0.1 \mathrm{M} \mathrm{KOH}$, as well as a very low $\mathrm{KOH}$ containing electrolyte denominated as $<0.001 \mathrm{M} \mathrm{KOH}$. In this last case the electrolyzer was supplied with de-gassed deionized water as feed solution, but a small amount of $1 \mathrm{M} \mathrm{KOH}$ is introduced into the system. The measured performance should thus not be directly compared to samples measured with pure water, as recently done successfully by Mayerhöfer et al. using Aemion, ${ }^{30}$ but is an indication for the trend expected to be seen at such low $\mathrm{KOH}$ concentrations.

A significant change in HFR and low current density kinetics can be observed for the $<0.001 \mathrm{M} \mathrm{KOH}$ feed solution, in stark contrast to the other samples, which show similar kinetics. The HFR up to above $600 \mathrm{~mA} \mathrm{~cm}{ }^{-2}$ follows the trend observed for Aemion by Fortin et al., ${ }^{\mathbf{1 6}}$ showing lower values for higher molarity of $\mathrm{KOH}$, but the difference shrinks for higher current densities of all $0.1,0.5$ and $1 \mathrm{M}$ feed solutions. The overall performance in this shown case follows previously reported trends from literature ${ }^{7,16}$ with the $1 \mathrm{M} \mathrm{KOH}$ outperforming $0.5 \mathrm{M}$ and $0.1 \mathrm{M} \mathrm{KOH}$ over the full current range, in that order. It should be noted that the clear order of the $0.1 \mathrm{M}$ and $0.5 \mathrm{M}$ measurements shown in this batch was not consistent over all repetitions in different spray batches, potentially indicating a more complex convolution of effects, possibly including sample-to-sample variations as well as optimized catalyst layers for a lower molarity feed solution. This could mean that ionomer content variations, similar to catalyst activity investigations, should ideally be directly performed for the intended final electrolyte or feed solution. ${ }^{31}$ Performance parameters (summarized in Fig. S10 $\dagger$ ) do not show trends outside of the observed error ranges for all measured samples, except for a clear increase in mass transport overpotential and HFR for the $<0.001 \mathrm{M} \mathrm{KOH}$ samples, which is in line with the data shown in Fig. S9.†

\section{Conclusions}

CCMs based on commercially-available, reinforced Aemion+ membranes and Aemion+ ionomer were fabricated and studied in water electrolysis operating in low molarity $\mathrm{KOH}$. The performance of the Aemion+ CCMs was comparable to reports for other AEMs $\left(1 \mathrm{~A} \mathrm{~cm}^{-2}\right.$ at under $\left.2 \mathrm{~V}\right)$, despite the catalyst loading being substantially reduced in comparison to previously reported CCS approaches $\left(1 \mathrm{mg}_{\text {Ir }} \mathrm{cm}^{-2}\right.$ vs. $3 \mathrm{mg}_{\text {Ir }} \mathrm{cm}^{-2}$ (ref. 16)) that relied on similar membranes. Using CCMs here allows an efficient utilization of catalyst material by ensuring a continuous catalyst/ionomer layer in direct contact with the membrane. With mechanically reinforced membranes also handling of the CCMs for AEM electrolysis greatly improved.

The morphology of the anode catalyst, $\mathrm{IrO}_{x}$, was studied and ionomer-dominated areas are identified in catalyst layers with a high ionomer content using cross-sectional SEM images, weakening the desired homogeneous pore space morphology in the catalyst layer. A variation of ionomer content in the cathode catalyst layer had no significant effect on the electrochemical cell performance. The ionomer content in the anode catalyst layers was varied determining the optimum in a range of ionomer contents between 4-15 wt $\%$. An anode ionomer content of $7 \mathrm{wt} \%$ provided the best compromise between performance and durability during short-term degradation tests at $1 \mathrm{~A} \mathrm{~cm}^{-2}$. A variation of feed solution by changing the molarity of $\mathrm{KOH}$ indicated that better performance is possible with the Aemion+ membrane in more strongly alkaline conditions, although this effect is most pronounced in very high or very low molarities.

Overall, the CCMs showed a comparable performance to other AEM-bases water electrolyzers. Future work may focus on ink stabilization and the development of ionomers as dedicated binders for homogenous and stable catalyst. The differences between the results shown in this study and other recent reports, ${ }^{19,25}$ however, also highlights the significant variations encountered when using different (experimental or commercially available) ionomers, membranes, fabrication methods and testing procedures.

\section{Conflicts of interest}

Steven Holdcroft is a co-founder of, and scientific advisor to, Ionomr Innovations Inc.

\section{Acknowledgements}

The authors would like to thank Ionomr Inc. for providing ionomer and membranes. The authors thank Hien Nguyen for assistance with XRF measurements, Philipp Veh and Carolin Klose for helpful input on CCM fabrication, and Joey Disch for fruitful discussions on electrolyzer testing systems. The authors gratefully acknowledge financial support by the Federal Ministry of Education and Research of Germany in the project FlexCoat (Grant No. 01DM19008A), the Natural Science and Engineering Research Council of Canada (NSERC) (Grant CRDPJ529968), and the Vector Stiftung within the project "CO2to-X".

\section{References}

1 K. Ayers, N. Danilovic, R. Ouimet, M. Carmo, B. Pivovar and M. Bornstein, Perspectives on Low-Temperature Electrolysis and Potential for Renewable Hydrogen at Scale, Annu. Rev. Chem. Biomol. Eng, 2019, 10, 219-239, DOI: 10.1146/ annurev-chembioeng-060718-030241.

2 M. R. Kraglund, M. Carmo, G. Schiller, S. A. Ansar, D. Aili, E. Christensen and J. O. Jensen, Ion-solvating membranes as a new approach towards high rate alkaline electrolyzers, Energy Environ. Sci., 2019, 36, 307, DOI: 10.1039/ C9EE00832B.

3 H. A. Miller, K. Bouzek, J. Hnat, S. Loos, C. I. Bernäcker, T. Weißgärber, L. Röntzsch and J. Meier-Haack, Green hydrogen from anion exchange membrane water 
electrolysis, Sustainable Energy Fuels, 2020, 4, 2114-2133, DOI: $10.1039 /$ C9SE01240K.

4 C. M. Gabardo, C. P. O'Brien, J. P. Edwards, C. McCallum, Y. Xu, C.-T. Dinh, J. Li, E. H. Sargent and D. Sinton, Continuous Carbon Dioxide Electroreduction to Concentrated Multi-carbon Products Using a Membrane Electrode Assembly, Joule, 2019, 3, 2777-2791, DOI: 10.1016/j.joule.2019.07.021.

5 J. Fan, S. Willdorf-Cohen, E. M. Schibli, Z. Paula, W. Li, T. J. G. Skalski, A. T. Sergeenko, A. Hohenadel, B. J. Frisken, E. Magliocca, W. E. Mustain, C. E. Diesendruck, D. R. Dekel and S. Holdcroft, Poly(bisarylimidazoliums) possessing high hydroxide ion exchange capacity and high alkaline stability, Nat. Commun., 2019, 10, 2306, DOI: 10.1038/s41467-019-10292-z.

6 C. Klose, T. Saatkamp, A. Münchinger, L. Bohn, G. Titvinidze, M. Breitwieser, K.-D. Kreuer and S. Vierrath, All-Hydrocarbon MEA for PEM Water Electrolysis Combining Low Hydrogen Crossover and High Efficiency, Adv. Energy Mater., 2020, 10, 1903995, DOI: 10.1002/ aenm.201903995.

7 H. Ito, N. Kawaguchi, S. Someya, T. Munakata, N. Miyazaki, M. Ishida and A. Nakano, Experimental investigation of electrolytic solution for anion exchange membrane water electrolysis, Int. J. Hydrogen Energy, 2018, 43, 17030-17039, DOI: 10.1016/j.ijhydene.2018.07.143.

8 Enapter, Enapter White Paper, 2020.

9 P. B. Alessandro Tampucci, Device for the production ondemand of hydrogen by electrolysis of aqueous solutions from dry cathode, 2009, EP2451992B1.

$10 \mathrm{~S}$. Holdcroft and J. Fan, Sterically-encumbered ionenes as hydroxide ion-conducting polymer membranes, Curr. Opin. Electrochem., 2019, 18, 99-105, DOI: 10.1016/ j.coelec.2019.10.014.

11 S. Gu, R. Cai, T. Luo, Z. Chen, M. Sun, Y. Liu, G. He and Y. Yan, A soluble and highly conductive ionomer for highperformance hydroxide exchange membrane fuel cells, Angew. Chem., Int. Ed. Engl., 2009, 48, 6499-6502, DOI: 10.1002/anie.200806299.

12 J. Wang, Y. Zhao, B. P. Setzler, S. Rojas-Carbonell, C. Ben Yehuda, A. Amel, M. Page, L. Wang, K. Hu, L. Shi, S. Gottesfeld, B. Xu and Y. Yan, Poly(aryl piperidinium) membranes and ionomers for hydroxide exchange membrane fuel cells, Nat. Energy, 2019, 4, 392-398, DOI: 10.1038/s41560-019-0372-8.

13 J. S. Olsson, T. H. Pham and P. Jannasch, Poly(arylene piperidinium) Hydroxide Ion Exchange Membranes, $A d v$. Funct. Mater., 2018, 28, 1702758, DOI: 10.1002/ adfm.201702758.

14 W.-H. Lee, E. J. Park, J. Han, D. W. Shin, Y. S. Kim and C. Bae, Poly(terphenylene) Anion Exchange Membranes, ACS Macro Lett., 2017, 6, 566-570, DOI: 10.1021/acsmacrolett.7b00148.

15 A. Lim, H.-j. Kim, D. Henkensmeier, S. Jong Yoo, J. Young Kim, S. Young Lee, Y.-E. Sung, J. H. Jang and H. S. Park, A study on electrode fabrication and operation variables affecting the performance of anion exchange membrane water electrolysis, J. Ind. Eng. Chem., 2019, 76, 410-418, DOI: 10.1016/j.jiec.2019.04.007.

16 P. Fortin, T. Khoza, X. Cao, S. Y. Martinsen, A. Oyarce Barnett and S. Holdcroft, High-performance alkaline water electrolysis using Aemion ${ }^{\mathrm{TM}}$ anion exchange membranes, $J$. Power Sources, 2020, 451, 227814, DOI: 10.1016/ j.jpowsour.2020.227814.

17 C. C. Pavel, F. Cecconi, C. Emiliani, S. Santiccioli, A. Scaffidi, S. Catanorchi and M. Comotti, Highly efficient platinum group metal free based membrane-electrode assembly for anion exchange membrane water electrolysis, Angew. Chem., Int. Ed. Engl., 2014, 53, 1378-1381, DOI: 10.1002/ anie.201308099.

18 I. V. Pushkareva, A. S. Pushkarev, S. A. Grigoriev, P. Modisha and D. G. Bessarabov, Comparative study of anion exchange membranes for low-cost water electrolysis, Int. J. Hydrogen Energy, 2020, 45(49), 26070-26079, DOI: 10.1016/ j.ijhydene.2019.11.011.

19 A. Y. Faid, L. Xie, A. O. Barnett, F. Seland, D. Kirk and S. Sunde, Effect of anion exchange ionomer content on electrode performance in AEM water electrolysis, Int. J. Hydrogen Energy, 2020, 45, 28272-28284, DOI: 10.1016/ j.ijhydene.2020.07.202.

20 H. Ito, N. Miyazaki, S. Sugiyama, M. Ishida, Y. Nakamura, S. Iwasaki, Y. Hasegawa and A. Nakano, Investigations on electrode configurations for anion exchange membrane electrolysis, J. Appl. Electrochem., 2018, 48, 305-316, DOI: 10.1007/s10800-018-1159-5.

21 A. G. Wright, J. Fan, B. Britton, T. Weissbach, H.-F. Lee, E. A. Kitching, T. J. Peckham and S. Holdcroft, Hexamethyl-p-terphenyl poly(benzimidazolium), Energy Environ. Sci., 2016, 9, 2130-2142, DOI: 10.1039/C6EE00656F.

22 T. Weissbach, A. G. Wright, T. J. Peckham, A. Sadeghi Alavijeh, V. Pan, E. Kjeang and S. Holdcroft, Simultaneous, Synergistic Control of Ion Exchange Capacity and CrossLinking of Sterically-Protected Poly(benzimidazolium)s, Chem. Mater., 2016, 28, 8060-8070, DOI: 10.1021/ acs.chemmater.6b03902.

23 M. Bühler, F. Hegge, P. Holzapfel, M. Bierling, M. Suermann, S. Vierrath and S. Thiele, Optimization of anodic porous transport electrodes for proton exchange membrane water electrolyzers, J. Mater. Chem. A, 2019, 7, 26984-26995, DOI: 10.1039/C9TA08396K.

24 European Commission. Joint Research Centre, EU harmonised polarisation curve test method for lowtemperature water electrolysis, Publications Office, 2018.

25 G. Huang, M. Mandal, K. Groenhout, A. Dobbs, N. Hassan, W. E. Mustain and P. Kohl, Ionomer Optimization for Water Uptake and Swelling in Anion Exchange Membrane Electrolyzer, J. Electrochem. Soc., 2020, 167(16), 164514, DOI: 10.1149/1945-7111/abcde3.

26 M. Bernt and H. A. Gasteiger, Influence of Ionomer Content in $\mathrm{IrO} 2 / \mathrm{TiO} 2$ Electrodes on PEM Water Electrolyzer Performance//Influence of Ionomer Content in IrO 2/TiO 2 Electrodes on PEM Water Electrolyzer Performance, $J$. Electrochem. Soc., 2016, 163, F3179-F3189, DOI: 10.1149/ 2.0231611jes. 
27 P. J. Rousseeuw and S. Verboven, Robust estimation in very small samples, Comput. Stat. Data Anal., 2002, 40, 741-758, DOI: $10.1016 /$ S0167-9473(02)00078-6.

28 T. Schuler, T. Kimura, T. J. Schmidt and F. N. Büchi, Towards a generic understanding of oxygen evolution reaction kinetics in polymer electrolyte water electrolysis, Energy Environ. Sci., 2020, 13, 2153-2166, DOI: 10.1039/ D0EE00673D.

29 F. Hegge, F. Lombeck, E. Cruz Ortiz, L. Bohn, M. von Holst, M. Kroschel, J. Hübner, M. Breitwieser, P. Strasser and S. Vierrath, Efficient and Stable Low Iridium Loaded Anodes for PEM Water Electrolysis Made Possible by Nanofiber Interlayers, ACS Appl. Energy Mater., 2020, 3, 8276-8284, DOI: 10.1021/acsaem.0c00735.
30 B. Mayerhöfer, D. McLaughlin, T. Böhm, M. Hegelheimer, D. Seeberger and S. Thiele, Bipolar Membrane Electrode Assemblies for Water Electrolysis, ACS Appl. Energy Mater., 2020, 3, 9635-9644, DOI: 10.1021/acsaem.0c01127.

31 J. Melder, P. Bogdanoff, I. Zaharieva, S. Fiechter, H. Dau and P. Kurz, Water-Oxidation Electrocatalysis by Manganese Oxides, Z. Phys. Chem, 2020, 234, 925-978, DOI: 10.1515/ zpch-2019-1491.

32 D. Li, A. R. Motz, C. Bae, C. Fujimoto, G. Yang, F. Y. Zhang, K. E. Ayers and Y. S. Kim, Durability of anion exchange membrane water electrolyzers, Energy Environ. Sci., 2021, 14, 3393-3419, DOI: 10.1039/D0EE04086J. 